\title{
The construction and quality assurance testing of the Fermilab Muon g-2 straw tracking detectors
}

\author{
Aaron Epps*广 \\ Northern Illinois University \\ E-mail: aeppsi@niu.edu
}

The Muon g-2 Experiment at Fermilab aims to measure the muon anomalous magnetic moment to a precision of 0.14 parts per million resulting in nearly a factor of four improvement on the previous measurement performed at Brookhaven National Lab. The straw tube tracking system provides an important contribution to this improvement. The tracking system will provide a measurement of the muon beam profile allowing for a reduction in systematic uncertainties and allow for pileup corrections in the calorimeter system. The tracking system will also allow for a search for the electric dipole moment of the muon.

Quality control in the tracking system is of particular importance, as the uncertainty in measurements taken by the tracking system will be determined by the quality of construction of the trackers. Important parameters include the position of the wire, the straw walls, and the gain. A test stand has been designed to measure these parameters. The test stand will scan across the tracking system utilizing a replaceable radioactive source.

38th International Conference on High Energy Physics

3-10 August 2016

Chicago, USA

${ }^{*}$ Speaker.
${ }^{\dagger}$ On behalf of the g-2 Collaboration

(c) Copyright owned by the author(s) under the terms of the Creative Commons 


\section{Introduction}

The g-2 experiment aims to measure the value of the anomalous magnetic moment of the muon to a precision four times greater than the previous experiment at Brookhaven National Laboratory(BNL) [1]. The experiment also aims to further constrain the current limit on the electric dipole moment. These improvements will be achieved through improved field measurements and upgrades in various systems resulting in reduced systematic uncertainties.

A quality control test stand has been designed and built to measure important parameters of the straw tube tracking system. The main goals of the system are to: ensure the functionality of each straw, accurately measure the gain, stereo angle, wire position, and straw wall position.

\section{2. g-2 Tracker System}

The g-2 tracker system consists of $5 \mathrm{~mm}$ diameter aluminized mylar tubes, filled with an equal part mixture of argon-ethane gas, with a $25 \mu \mathrm{m}$ gold plated wire at the center. These straws are arrayed in four, thirty-two-straw layers in a tracker module. Each layer is at a $7.5^{\circ}$ angle to the vertical. Trackers are to be placed at three locations around the g- 2 ring with 8 trackers at each location.

Reconstructed tracks allow for measurement of the beam profile and a reduction in pileup uncertainty in the calorimeter. They also allow for a measurement of precession plane tilt which is the main source of sensitivity to an electric dipole moment. The improved system provides a reduction in systamatics of $0.18 \mathrm{ppm}$ and a $4 \mathrm{um}$ improvement in precession plane tilt over the measurment performed at BNL [1].

\section{Mechanical Design}

The test stand consists of a frame in which a tracker can be secured. Mounted on the frame are two movable stages that allow for $6 \mu \mathrm{m}$ steps in both the horizontal and vertical. Attached to the stages is a horseshoe arm that allows mounting of a scintillator and silicon photomultiplier(SiPM) on one side and a radioactive source on the other. The source is replaceable and both a Fe-55 and Sr-90 source are used in the stand.

\section{Sr-90 Measurements}

Measuring the wire position within a straw is accomplished by making a measurement of the relative time between a straw hit from an electron and the corresponding measurement from the SiPM. As the distance from the center of the wire increases the drift distance of the ionization produced increases, and so does the relative time between the two hits. Horizontal scans will be performed across the entire tracker at a number of vertical positions. These scans can be combined to make a determination of the straw angle. Simulations were performed to determine the optimal parameters to use in performing this scan. Accuracy in determing wire position and time taken to complete a scan are competing figures of merit in the process.

Garfield, a gaseous detector simulator, was used to obtain position and time information for fifteen million electrons passing through a straw with the wire both at the origin and shifted to other 


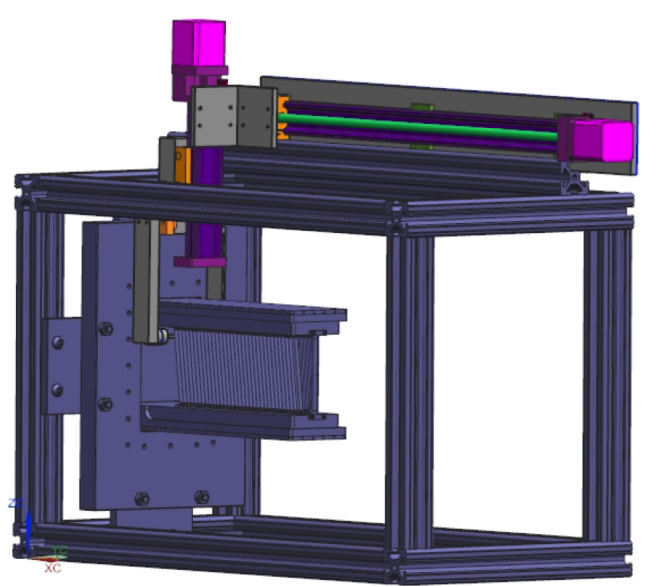

Figure 1: Engineering Drawing of Test Stand

positions [2]. This data was used to simulate scans at a varying number of hits per position, number of positions, collimator widths, and fitting methods. Figure 2 shows an overlay of raw x-t data, the raw data binned, and the fit. The results of multiple fits were then combined into residual plots.
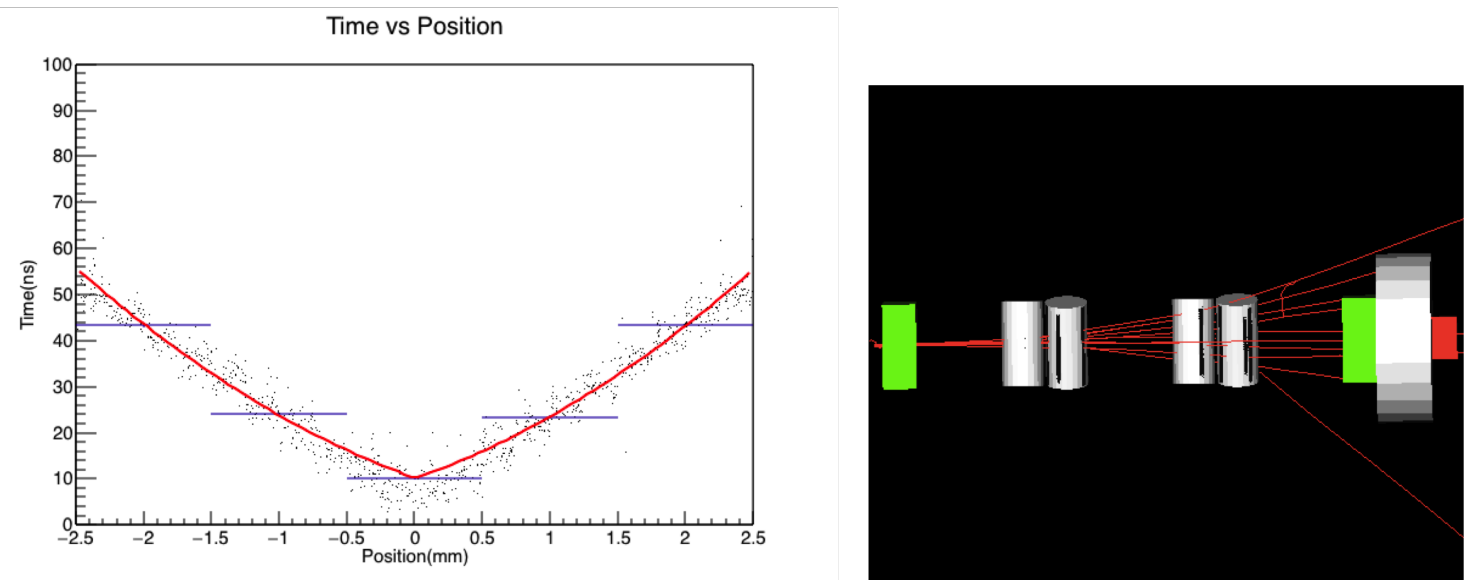

Figure 2: Left: Overlay of Raw Data(black points), Binning(points with blue horizontal error bars), and Fit(red line) Right: G4beamline Simulation

G4beamline was used to simulate the effect of collimator size and multiple scattering, ultimately leading to a estimate of the time neccessary to complete a scan [3]. Most electrons that would otherwise make it through the system are lost to scattering.

The result of the simulations led to the choice of a $1 \mathrm{~mm}$ by $3 \mathrm{~mm}$ collimator. The optimal fit was determined to be a symmetric second degree polynomial. The horizontal wire position can be determined to better than $10 \mu \mathrm{m}$. The wire angle can be determined to better than $100 \mu \mathrm{rad}$. These parameters can be determined with a scan that takes less than 24 hours.

\section{Fe-55 Measurements}

Fe-55 allows for the use of a much smaller collimator as multiple scattering is not a large 
effect. This makes Fe-55 the optimal source to use in measuring the position of the straw walls. Scanning with Fe-55 will be performed with a $25 \mu \mathrm{m}$ circular collimator. Monte Carlo combined with G4beamline simulations result in an effective rate of $20 \mathrm{~Hz}$ for this collimator. Much less data is needed to complete this scan, but further studies are neccessary.

Fe-55 is also essentially monoenergetic allowing for an accurate determination of the gain. When a photon is absorbed by a straw it deposits a known amount of energy. The gain measurement will be performed at one position in each straw.

A separate scan to determine straw functionality is unneccesary as a broken straw will become apparant in any of the other measurements.

\section{Conclusion}

This test stand will provide an efficient and repeatable way to measure important design parameters of the straw tube trackers. Construction of the test stand is complete and initial testing to confirm simulation is now underway. Testing of the trackers will commence as they arrive starting in Fall 2016.

\section{Acknowledgments}

I would like to thank my advisor Michael Eads. This work was supported in part by the US DOE (DOE Award DE-SC0012231) OHEP and ONP, the US NSF, the UK STFC, the NNSF of China and by INFN in Italy.

\section{References}

[1] Muon G-2 collaboration, J. Grange et al., Muon (g-2) Technical Design Report, 1501.06858.

[2] R. Veenhof, "Garfield - simulation of gaseous detectors." online at http://cern.ch/garfield.

[3] “G4beamline.” http://g4beamline.muonsinc.com. 